SNA + MC 2013, $05128(2014)$

DOI: $10.1051 /$ snamc/2014 05128

(c) Owned by the authors, published by EDP Sciences, 2014

\title{
Performance Assessment of the Commercial CFD Software for the Prediction of the PWR Internal Flow - Corrected versiona
}

\author{
Gong Hee Lee ${ }^{1^{*}}$, Young Seok Bang ${ }^{1}$, Sweng Woong Woo ${ }^{1}$, Ae Ju Cheong ${ }^{1}$, Do Hyeong Kim ${ }^{2}$, Min Ku Kang ${ }^{2}$ \\ ${ }^{1}$ Korea Institute of Nuclear Safety, Daejon, Republic of Korea \\ ${ }^{2}$ ANFLUX Inc., Seoul, Republic of Korea \\ ${ }^{*}$ Corresponding Author, E-mail: ghlee@kins.re.kr
}

\begin{abstract}
As the computer hardware technology develops the license applicants for nuclear power plant use the commercial CFD software with the aim of reducing the excessive conservatism associated with using simplified and conservative analysis tools. Even if some of CFD software developers and its users think that a state of the art CFD software can be used to solve reasonably at least the single-phase nuclear reactor safety problems there is still the limitations and the uncertainties in the calculation result. From a regulatory perspective, Korea Institute of Nuclear Safety (KINS) has been presently conducting the performance assessment of the commercial CFD software for the nuclear reactor safety problems. In this study, in order to examine the prediction performance of the commercial CFD software with the porous model in the analysis of the scale-down APR + (Advanced Power Reactor Plus) internal flow, simulation was conducted with the on-board numerical models in ANSYS CFX R.14 and FLUENT R.14. It was concluded that depending on the CFD software the internal flow distribution of the scale-down APR+ was locally some-what different. Although there was a limitation in estimating the prediction performance of the commercial software due to the limited number of the measured data, CFX R.14 showed the more reasonable predicted results in comparison with FLUENT R.14. Meanwhile, due to the difference of discretization methodology, FLUENT R.14 required more the computational memory than CFX R.14 for the same grid system. Therefore the CFD software suitable to the available computational resource should be selected for the massive parallel computation.
\end{abstract}

KEYWORDS: Flow Similarity, Porous Assumption, Reactor Internal Flow, Turbulent Flow

\begin{abstract}
${ }^{a}$ This corrected version replaces the article Performance Assessment of the Commercial CFD Software for the Prediction of the Reactor Internal Flow, SNA + MC 2013 - Joint International Conference on Supercomputing in Nuclear Applications + Monte Carlo 05106 (2014), DOI: 10.1051/snamc/201405106, which has been formally withdrawn.
\end{abstract}

\section{Introduction}

Because the design change of a reactor may influence thermal hydraulic characteristics inside a reactor, licensing applicants conduct a flow distribution test and use test results as the input data for the core thermal margin analysis program. Although the competitiveness of CFD is continuously growing due to the rapid developments in computer hardware technology, computer capacity is still a limiting factor for CFD calculations to produce completely accurate results in the prediction of reactor internal flow. Therefore the simplified geometries and turbulence models may result in modeling errors and numerical errors that give more or less inaccurate results. In this study, in order to examine the prediction performance of the commercial CFD software with the porous model in the analysis of the scale-down APR+ internal flow, simulation was conducted with the on-board numerical models in ANSYS CFX R.14 $4^{(1)}$ and FLUENT R.14 ${ }^{(2)}$.

\section{Analysis Model}

1. APR+ flow distribution test facility

APR + Core Flow \& Pressure Test Facility (ACOP) is a $1 / 5$ scale model of APR+ and consists of a reactor pressure vessel with four inlet (cold leg) and two outlet nozzles (hot leg). ${ }^{(3)}$

\section{Test conditions}

The test matrix consists of the symmetric/asymmetric flow conditions for four-pump operation and the flow conditions for three-pump operation. In this study, CFD simulation with the symmetric flow conditions for four-pump operation was conducted. In this condition, the Reynolds number was about $8.6 \times 10^{5}$ in the downcomer.

\section{Geometry modeling}

The internal structures, especially located in the upstream of reactor core, may have a significant influence on the core inlet flow rate distribution depending on both their shapes and the relative distance between the internal structures and the core inlet. Therefore the exact representation of these internal structures is needed for the core inlet flow rate distribution. However, such an approach requires much more computation resource to analyze the real flow phenomena inside a reactor.

In this study, as shown in Figure 1, the real geometry of flow skirt and beams in the lower support structure were considered. On the other hand, fuel assembly and some internal structures, for examples instrument nozzle support, fuel alignment plate, and upper plenum were considered as 
each simple bulky volume (porous domain) due to the limitation of computation resource. Then, in order to reflect the velocity field and pressure drop occurring in the original flow region, porosity and isotropic loss model were applied to porous domain.

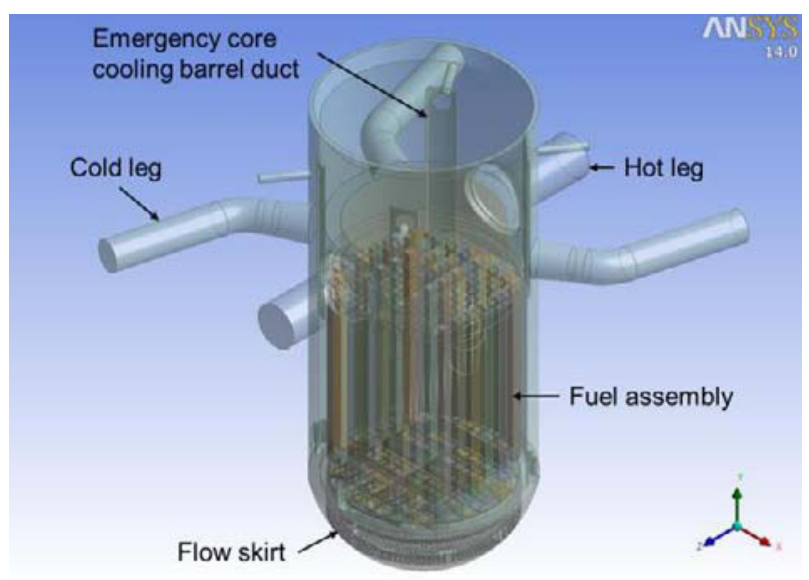

Figure 1: The computational domain.

\section{Numerical Modeling}

\section{Numerical method}

The flow inside the scale-down APR+ model was assumed to be steady, incompressible and isothermal. High resolution scheme in CFX and the second order upwind scheme in FLUENT were used for the convection terms of momentum equations. Meanwhile, to enhance the convergence the first order upwind scheme was used for the convection terms of turbulence equations. The solution was considered to be converged when the residuals of variables were below $4 \times 10^{-4}$ and the variations of the target variables were small.

\section{Turbulence model}

Standard k- $\varepsilon$ model, which is one of Reynolds-averaged Navier-Stokes (RANS)-based turbulence models, was used to simulate the turbulent flow inside the scale-down APR+.

\section{Grid system}

A hybrid mesh, made up of tetrahedron and prism, was generated to improve mesh resolution in the near wall region. Total numbers of grid were $6.3 \times 10^{7}$ and maximum $y+$ in the downcomer was about 305 . This grid system was determined from the grid sensitivity study. ${ }^{(4)}$

\section{Boundary conditions}

Inlet flow rate of $135 \mathrm{~kg} / \mathrm{s}$ was imposed at each cold leg. Turbulence intensity at inlet was assumed to be $5 \%$. Light water of $60^{\circ} \mathrm{C}$ was used as the working fluid. Average pressure over whole outlet option with the relative pressure of $0 \mathrm{~Pa}$ was used at each hot leg as an outlet boundary condition. No-slip condition was applied on the solid wall.

\section{Results and Discussion}

Depending on the CFD software the internal flow distribution of the scale-down APR+ was locally somewhat different. The measured core inlet flow rate was in the range of $86 \%$ to $126 \%$ of the averaged fuel assembly flow rate. Both CFX and FLUENT predicted core inlet flow rate in the range of $41 \%$ to $145 \%$ and $26 \%$ to $154 \%$ respectively. Although there was a difference between the measurement and the prediction, CFX showed more agreement with the measurement in comparison with FLUENT.

Figure 2 shows the frequency distribution of the mass flow rate at core inlet plane. Standard deviation of mass flow rate $(\sigma)$ in CFX was smaller than that in FLUENT. This means that CFX may predict the more uniform distribution of mass flow rate at core inlet plane. Meanwhile FLUENT predicted the wider minimum/maximum distribution of the mass flow rate at core inlet plane.

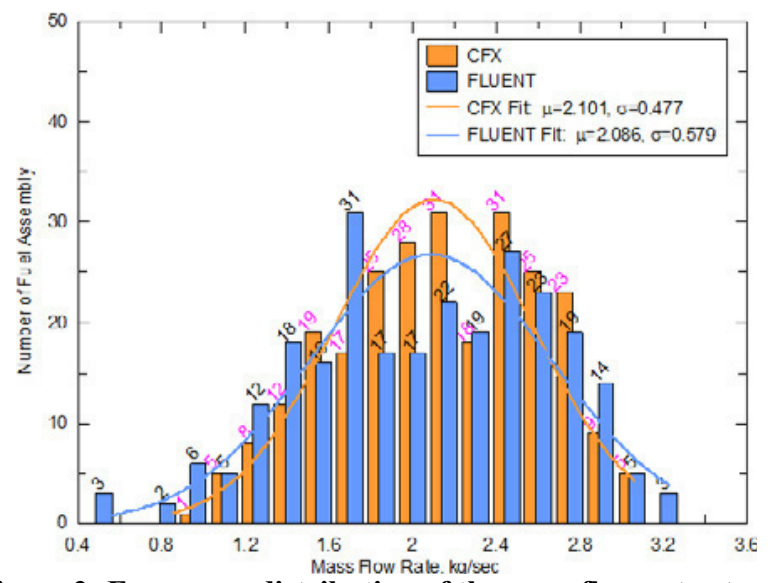

Figure 2: Frequency distribution of the mass flow rate at core inlet plane

Because of the difference of discretization methodology, FLUENT R.14 (node based discretization scheme) required more the computational memory than CFX R.14 (cell based discretization scheme) for the same grid system. Therefore CFD software suitable to the available computational resource should be selected for the massive parallel computation.

\section{Acknowledgment}

This work was conducted under the financial support of the Nuclear Safety and Security Commission of Korea [project title: Development of Regulatory Evaluation Technologies for Thermal-hydraulic Safety]. This work was also supported by the Supercomputing Center/Korea Institute of Science and Technology Information with supercomputing resources including technical support [project number: KSC-2013-C1010].

\section{References}

1) ANSYS CFX, Release 14.0, ANSYS Inc.

2) FLUENT, Release 14.0, ANSYS Inc.

3) D.J. Euh et al., "A Flow and Pressure Distribution of APR+ Reactor under the 4-Pump Running Conditions with a Balanced Flow Rate," Nuclear Engineering and Technology, 44, 735-744 (2012).

4) G.H. Lee et al., "A Numerical Study for the Effect of Flow Skirt Geometry on Reactor Internal Flow," Annals of Nuclear Energy, 62, 452-462 (2013). 